\title{
Apoio Matricial em Saúde Mental: revisão narrativa do uso dos conceitos horizontalidade e supervisão e suas implicações nas práticas
}

\author{
Matrix support in Mental Health: narrative revision of the concepts \\ horizontality and supervision and their practical implications
}

Luiz Fernando Chazan (https://orcid.org/0000-0001-8928-2240) ${ }^{1}$

Sandra Lucia Correia Lima Fortes (https://orcid.org/0000-0002-9918-1555) ${ }^{1}$

Kenneth Rochel de Camargo Junior (https://orcid.org/0000-0003-3606-5853) ${ }^{2}$
${ }^{1}$ Faculdade de Ciências Médicas, Universidade do Estado do Rio de Janeiro. A. Prof. Manoel de Abreu 444, Vila Isabel. 20550-170 Rio de Janeiro RJ Brasil. lfchazan@gmail.com

${ }^{2}$ Instituto de Medicina Social, Universidade do Estado do Rio de Janeiro. Rio de Janeiro RJ Brasil.

\begin{abstract}
The Matrix Support (MS) is one of the cornerstones of the integration between Primary Health Care (PHC) professionals and Mental Health professionals (MH). A narrative review was conducted on the articles on MS in MH published in national databases from 1998 to 2017, considering a brief history of the $P H C$ reorganization processes that led to the creation of the MS proposal. The aim was to understand the meanings attributed to the terms "horizontality" and "supervision" as well as the descriptions of the "matrix support" itself. We sought to identify factors contributing to the difficulties that have been described in the practices and literature, based on the assumption that these concepts are polysemous and it is possible to generate ambiguities that operate to the detriment of interprofessional practices. Based on the analysis of the selected articles, we were able to conclude that, in addition to polysemy, the obstacles' force lies in the hegemonic model of professional Health training, as it is traditional, hierarchical and uni-professional, and hinders the development of dialogic relations that favor the integration of the matrix support teams and PHC and consequent resolubility and quality of care.
\end{abstract}

Key words Matrix Support, Mental Health, Primary health care, Horizontality, Supervision
Resumo O Apoio Matricial (AM) é considerado um dos pilares da integração entre os profissionais da Atenção Primária à Saúde (APS) e os profissionais de Saúde Mental (SM). À luz de um breve histórico dos processos de reorganização da APS que levaram à criação da proposta de AM e de considerações sobre seus conceitos fundantes, realizou-se uma revisão narrativa dos artigos sobre AM em SM publicados nas bases de dados nacionais de 1998-2017. Procurou-se compreender os sentidos atribuidos aos termos "horizontalidade" $e$ "supervisão", assim como as descrições de AM. Buscou-se identificar fatores contribuintes para as dificuldades que têm sido descritas nas práticas, partindo-se do pressuposto de que estes conceitos são polissêmicos e podem gerar ambiguidades que operam prejudicando as ações interprofissionais. Dos 106 artigos encontrados, 39 citaram o termo "horizontalidade' e 29 citaram "supervisão". Na análise destes textos concluiu-se que, para além da polissemia, os obstáculos têm sua força no modelo ainda hegemônico de formação profissional em Saúde, tradicional, hierárquico e uni-profissional dificultando o desenvolvimento de relações dialógicas que favoreçam a integração das equipes de apoio matricial e da APS e consequente resolubilidade e qualidade assistencial.

Palavras-chave Apoio Matricial, Saúde Mental, Atenção Primária, Horizontalidade, Supervisão 


\section{Introdução}

A integração de cuidados em Saúde Mental e dos cuidados na Atenção Primária à Saúde é considerada como prioritária pela Organização Mundial de Saúde, dada a carga mundial de transtornos mentais e as lacunas de assistência neste campo.

No contexto internacional "Cuidados Colaborativos" ou ainda "Cuidados Compartilhados" ${ }^{\prime \prime 2}$ são denominações de uma prática que integra profissionais de Saúde Mental (pSM) e profissionais de Atenção Primária (pAPS), visando a garantia de atendimento integral das necessidades de saúde da população.

No Brasil, para compreender como essas denominações se integram, é necessário fazer um breve resgate de como se deu a conformação da APS no país, com destaque para o final da década de 1980 e início dos anos 1990, período marcado pela Constituinte que consagrou saúde como um direito do cidadão e um dever do Estado, bem como concretizou a pauta do movimento da reforma sanitária brasileira para criação de um Sistema Único de Saúde (SUS). Em 1994, tendo em vista o acúmulo de experiências já em curso no país, no campo da APS e baseado nos princípios do SUS, integralidade, universalidade e equidade, o governo criou o Programa Saúde da Família (PSF), que em seu escopo contava com uma equipe formada por médicos, enfermeiros e agentes comunitários de saúde. Dessa forma, novas necessidades se apresentaram, em especial as relacionadas à formação dos profissionais, considerada insuficiente para a prática da assistência centrada no usuário, como previsto pela APS $^{3-6}$.

No âmbito da Saúde Mental, em 1997 Adib Janete e Davi Capistrano propuseram a criação do Projeto Qualis/PSF, que consistia em equipes de Saúde Mental com o objetivo de produzir alterações na estrutura assistencial e gerencial dos serviços de saúde, oferecendo suporte técnico às Equipes de Saúde da Família ${ }^{7}$. Este projeto é considerado o embrião das propostas subsequentes neste campo ${ }^{8}$.

Em 1999, Gastão Wagner de Sousa Campos, buscando adequar a realidade do arranjo organizacional da saúde no país, cunhou o termo "apoio especializado matricial", hoje conhecido como "Apoio Matricial"10 (AM) que passou a compor o rol de recomendações oficiais ${ }^{11,12}$ para atuação dos Núcleos de Apoio à Saúde da Família (NASF) com as Equipes de Saúde da Família (eSF)

O AM aparece como uma diretriz para a inclusão de ações em Saúde Mental na APS em "Saúde Mental e Atenção Básica - O vínculo e o diálogo necessários"13 do Ministério da Saúde de 2003, porém somente em 2008 com a criação do Núcleo de Apoio à Saúde da Família (NASF) ${ }^{14}$, o AM passa a ser prática efetiva nas Unidades de Saúde da Família, que passa a ter em seu escopo profissionais de vários campos da saúde, variando a sua composição em função de características regionais, dependendo das gestões municipais, exceção para os profissionais de Saúde Mental que são participantes obrigatórios dos Núcleos.

As Diretrizes dos NASF ${ }^{11}$ destacam a importância do trabalho integrado entre os profissionais de SM e Saúde da Família uma vez que isso potencializaria o cuidado na perspectiva de uma abordagem integral de saúde. O NASF seria, portanto, o principal dispositivo de atenção à saúde mental no âmbito da atenção primária à saúde ${ }^{15}$, o que evidencia a importância do trabalho com base no AM, tendo em vista que os Núcleos têm nele sua principal ferramenta de atuação.

O objetivo do AM seria a corresponsabilidade no cuidado em saúde entre equipes multiprofissionais da APS e profissionais apoiadores especialistas, de maneira que as relações sejam horizontais e a troca de saberes não seja hierarquizada $^{16,17}$.

Entre as ações previstas pelo Apoio Matricial na APS destacam-se as consultorias técnico-pedagógicas, os atendimentos conjuntos e as ações assistenciais específicas, que devem ser sempre dialogadas com a equipe de referência. A assistência individual, temporalmente limitada, também é possível ${ }^{18}$ mantendo-se a corresponsabilidade entre APS e AM. A concepção do AM reconhece que nenhum profissional isolado pode garantir o cuidado integral à saúde ${ }^{19}$.

O conceito AM é composto por duas dimensões que integram os atores (apoio) e a organização dos serviços (matricial). O termo "apoio" pressupõe relações entre sujeitos, diálogo, interdisciplinaridade e relações horizontais e "matricial", a organização de uma rede de cuidado integral, formada a partir do diálogo e objetivando a assistência longitudinal. AM é um arranjo organizacional e um modo de trabalho interprofissional $^{9,20}$.

Diante disso seria possível apontar o que é indispensável para se conceber como uma boa prática de AM? Sua essência? Com base em Campos $^{9,21}$ e Campos e Domitti ${ }^{22}$ e nos Cadernos de Atenção Básica ${ }^{11,12,23}$, o apoio matricial pode promover a redistribuição do poder através de uma gestão horizontal e colegiada.

Este sistema matricial que combina referência (trabalho mais polivalente) com oferta horizontal 
(trabalho mais específico e especializado) permite valorizar todas as profissões de saúde, tanto conservando a identidade de cada uma delas, quanto empurrando-as para superar uma postura muito burocratizada, típica do modo tradicional de organizar os serviços de saúde $e^{9}$ (p.398).

Desta forma as relações interprofissionais tenderiam ao diálogo, base de uma prática democrática. Assim, as equipes de referência e de apoio matriciais em parceria com os pacientes poderiam alcançar uma Clínica Ampliada ${ }^{24}$ voltada para o sujeito e suas necessidades. Através das práticas com este embasamento o desenvolvimento de comunicação eficaz e eficiente permite o aprimoramento da educação permanente, com benefícios para a corresponsabilização, prevenção e integralidade do cuidado ao longo do tempo.

As equipes de AM têm sua razão de ser na relação com os profissionais da APS e suas tarefas e, assim, pode-se reforçar o papel da horizontalidade nas relações como elemento fundamental.

Apesar da bibliografia citada, são descritas dificuldades nas práticas do AM como citado por Costa et al. ${ }^{19}$ :

A análise sobre como circulam os saberes entre especialistas e equipe de referência nos depoimentos permitiu concluir que há um distanciamento entre intenção e atuação nas diferentes formas de operar o AM. Nos exemplos relatados de AM, notou-se uma forte presença de princípios liberais ou tradicionais, ao subsidiar suas ações na transmissão do saber como o modo pelo qual as pessoas adquirem conhecimento. Para alguns profissionais, o reconhecimento da necessidade de atuarem de uma forma dialógica emergiu como tensão e desejo, deixando evidente a falta de ferramentas pedagógicas para atuarem de maneira diversa à prática revela$d a^{19}$ (p.498).

Dadas as dificuldades, no Guia Prático de Matriciamento $^{2}$ apresenta-se o que o AM não é:

- encaminhamento ao especialista;

- atendimento individual pelo profissional de saúde mental;

- intervenção psicossocial coletiva realizado apenas pelo profissional de saúde mental.

O Guia² afirma que:

O matriciamento deve proporcionar a retaguarda especializada da assistência, assim como um suporte técnico-pedagógico, um vínculo interpessoal e o apoio institucional no processo de construção coletiva de projetos terapêuticos junto à população. Assim, também se diferencia da supervisão, pois o matriciador pode participar ativamente do projeto terapêutico²(p.14-15).
Torna-se, portanto, relevante refletir sobre os possíveis elementos que contribuem para que as práticas não atendam ao proposto. Podendo-se incluir nesta categoria, conceitos polissêmicos que podem ser entendidos com o viés do profissional praticante.

Nesse sentido, o presente artigo tem como objetivo central analisar o conceito de horizontalidade apresentado na literatura sobre o Apoio Matricial no campo da Saúde Mental, entendido como o campo de cuidado às pessoas com sofrimento psíquico, portadoras ou não de transtornos mentais ${ }^{2}$. Como objetivos secundários, propõem-se uma análise dos usos do termo Supervisão cotejando com as diferentes acepções utilizadas nos principais trabalhos do campo $^{9,10,20-22}$ e como o conceito de Apoio Matricial ou Matriciamento são citados.

\section{Método}

Trata-se de uma revisão narrativa ou tradicional compreendida como uma metodologia qualitativa que fornece uma base para validar premissas, e compreensão de outros estudos estimulando pensamentos e controvérsias ${ }^{25}$. Rother ${ }^{26}$ esclarece que estas são revisões "apropriadas para descrever e discutir o desenvolvimento ou o "estado da arte" de um determinado assunto, sob ponto de vista teórico ou contextual". Este tipo de revisão critica e resume conclusões sobre o tópico em questão, utilizando-se de estudos e conhecimentos relevantes sobre o tema. A revisão narrativa não prevê, necessariamente, a explicitação dos critérios utilizados no processo de seleção dos materiais, o que não significa que não há nenhuma regra determinada pelos pesquisadores. É útil para resumir e sintetizar uma área específica assim como tem seu papel na educação continuada sendo essencialmente qualitativa ${ }^{5,27}$. A pesquisa foi realizada nas bases de dados da Biblioteca Virtual em Saúde (BVS) com o recorte temporal de 1998 a 2017. O processo de seleção do material foi realizado em duas etapas, a primeira foi uma busca utilizando os termos "apoio matricial" ou "matriciamento" e "saúde mental". Foram pesquisados somente artigos nacionais, pois o Apoio Matricial, como proposto por Gastão Wagner de Souza Campos, foi criado no contexto da realidade brasileira. A segunda etapa consistiu na busca dos termos "horizontalidade" e/ou "supervisão" para filtrar dentre os artigos já selecionados. Aqueles que não apresentavam nenhum desses termos em seus conteúdos foram excluídos. Só 
foram incluídos artigos com textos completos. Os artigos duplicados foram considerados unitariamente. Todos foram investigados em busca das definições dos conceitos de Horizontalidade, Supervisão e Apoio Matricial ou Matriciamento.

\section{Resultados e Discussão}

Foram encontrados no período de 1998 e 2017, 106 artigos sobre Saúde Mental com o termo Apoio Matricial e ou com o termo Matriciamento. Após a exclusão dos artigos que não mencionaram os termos Horizontalidade e/ou Supervisão restaram 83, listados no Quadro 1.

\section{Sobre Apoio Matricial}

Não se observou diferenças no uso de Apoio Matricial ou Matriciamento. Os termos são usados indistintamente e até declaradamente como sinônimos ${ }^{28}$.

Não se trata aqui de discutir os conceitos pressupondo uma verdade universal, mas sim tomar a proposta dialógica AM/ESF como parâmetro definidor do que se necessita para seu êxito. Ao se utilizar "matriciamento" como sinônimo de "apoio matricial" simplifica-se a proposta original? Partindo dos textos em análise os termos "apoio" e "matricial" são operadores da proposta e complementares ${ }^{22}$.

Percebe-se que a margem dada às diferentes interpretações destes conceitos, pela polissemia que os caracteriza, tem sido parte dos obstáculos citados por Campos ${ }^{22}$. Ainda com base nos materiais selecionados, é possível identificar que os conceitos traduzem um "modus operandi", e que nas entrelinhas suscitam interpretações diversas, não menos determinante das práticas.

O cuidado para não fetichizar ${ }^{29}$ as palavras é fundamental, como se fosse possível separar os sentidos e significados dos sujeitos que as pensam.

Os conceitos fundamentais para a prática constituem uma rede que dá sustentação para a dinâmica de trabalho. Um conceito mal definido ou compreendido pode ampliar a fragilidade da rede, que pode ser prevenida através do diálogo, uma vez que é através dele que as mudanças são possíveis, ampliando visões acerca de novos sentidos e significados.

Apesar do conceito de Apoio Matricial ser comumente utilizado e compor as propostas que visam integrar o cuidado em SM na APS, há questões em aberto, que contribuem para refletir a respeito do sucesso ou não do processo de criação de assistência de qualidade e de educação permanente.

A primeira questão diz respeito à própria compreensão da proposta, que como afirma Fittipaldi et al. ${ }^{15}$, o AM foi inserido como política pública sem que houvesse qualificação dos profissionais para o caráter inovador dessa metodologia de trabalho. Castro e Campos ${ }^{16}$ reforçam essa percepção, observando que as resistências a esse projeto são fruto do desconhecimento da maioria dos profissionais sobre o AM, e quando conhecem, têm dificuldades no entendimento e na aplicação desse método. Pode-se supor que se há pouca clareza na proposta do $\mathrm{AM}$, os profissionais tenderão a funcionar no seu modelo familiar, que no caso da SM é a supervisão. Desta forma se reforça a confusão conceitual, pois o Apoio Matricial, refere-se a um arranjo organizacional em que duas equipes se apoiam mutuamente e horizontalmente para levarem à assistência na APS a qualidade resolutiva esperada.

As compreensões encontradas de AM são heterogêneas, o que resulta em um uso genérico da expressão, podendo ser atribuído a qualquer prática dos profissionais responsáveis por esse trabalho. Alguns estudos, inclusive, sugerem que

Quadro 1. Relação dos artigos.

\begin{tabular}{|l|c|l|}
\hline \multicolumn{1}{|c|}{ Tema } & $\begin{array}{c}\text { Quantitativo } \\
\text { dos artigos } \\
\text { selecionados }\end{array}$ & Referência dos artigos selecionados para análise \\
\hline Contém Horizontalidade & 39 & $\begin{array}{l}1,3,6,9,16,19,21,22,28,30,36,37,40,42,47,48, \\
50,51,52,53,54,55,56,57,58,59,60,61,62,63, \\
64,65,66,67,68,69,70,71,72,73\end{array}$ \\
\hline Contém Supervisão & & $\begin{array}{l}1,6,9,17,21,30,38,40,41,42,44,47,51,61,62, \\
65,67,69,70,71,74,75,76,77,78,79,80,81\end{array}$ \\
\hline Contém Horizontalidade e Supervisão & 28 & $1,6,9,21,30,40,44,47,51,61,62,65,67,69,70,71$ \\
\hline
\end{tabular}

Fonte: elaboração própria. 
os trabalhadores não têm clareza do que AM significa ${ }^{8,30}$

\section{Sobre horizontalidade}

Chama atenção, que dos 106 artigos, 51 não citem horizontalidade, apesar da sua participação estrutural na proposta do AM.

Através da leitura dos artigos selecionados que tratam do tema da horizontalidade é possível classificá-la, em função do significado a ela atribuída, em 6 categorias como demonstrado no Quadro 2.

Reflexões acerca do significado do termo são fundamentais para a sua aplicabilidade. Algumas contribuições realizadas no contexto da I Conferência Nacional de Saúde Mental versam sobre a importância da horizontalização das relações de trabalho entre os profissionais, como algo fundamental para que a construção do vínculo entre os profissionais e os usuários do Sistema de Saúde não aconteça de forma verticalizada ${ }^{31}$.

A horizontalidade pode ser definida como uma relação entre sujeitos, em que o saber por si, não define hierarquias e é, portanto, condição para o diálogo, para tanto pode-se compreender o diálogo como uma necessidade existencial que amalgama a reflexão e a ação, superando as simples trocas ou depósitos de ideias em outrem ${ }^{32}$. Desta forma a proposta inter e transdisciplinar do AM é sustentada na horizontalidade, no diálogo e na compreensão de que conhecimentos diferentes não estabelecem hierarquias, mas sim complementaridades.

No jogo do poder dos serviços, a verticalidade e a horizontalidade concorrem. A verticalidade se constitui no poder do saber de um sobre outro, enquanto a horizontalidade atua na distribuição do poder que encontra na coparticipação seu ápice, modificando e construindo realidades ${ }^{33}$.

A horizontalidade permite reduzir o modelo hierárquico professor-aluno e estimular o trabalho colaborativo em que um "saber" não vale mais que outro, principalmente se isolado em si próprio. Um exemplo simples é o da relação do profissional de saúde e do paciente em que se contrapõem dois "saberes", o "saber" do profissional (conhecimento técnico, formal) e o "saber" do paciente (conhecimento sobre si e suas circunstâncias), que precisam interagir para criar vínculo e confiança. Os dois precisam assumir que nenhum dos saberes em questão se bastam por si, e que só através de um reconhecimento mútuo e integrativo é que será possível caminhar para um desfecho em direção à saúde. Como nos diz Barreto ${ }^{34}$ : "não existe um saber superior ao outro, mas um saber a compartilhar. Todos somos aprendizes".

Podemos, portanto, entender que a horizontalidade contribui para a constituição de boas relações de trabalho que geram modelos dialógicos e que essa experiencia reverbera na relação ESF/ Paciente/Família.

Esta horizontalidade trilateral ${ }^{9}$, constituída por profissionais da SF, do AM e usuários, é especialmente importante, pois o êxito da proposta do AM não está somente no âmbito das relações profissionais, mas também na relação do usuário com o serviço de quem se espera corresponsabilidade também trilateral.

Parceria, colaboração e reciprocidade são fluídas onde a horizontalidade se faz presente. Não há expectativa de uniformidade, mas sim de diferenças, respeitadas e valorizadas entre pessoas,

Quadro 2. Distribuição dos artigos por categorias de análise.

\begin{tabular}{|l|c|l|}
\hline \multicolumn{1}{|c|}{ Categorias } & $\begin{array}{c}\text { Quantitativo } \\
\text { dos artigos } \\
\text { selecionados }\end{array}$ & \multicolumn{1}{|c|}{$\begin{array}{c}\text { Referência dos artigos selecionados } \\
\text { para análise }\end{array}$} \\
\hline $\begin{array}{l}\text { Organização dos serviços/democracia/ } \\
\text { distribuição do poder }\end{array}$ & 19 & $\begin{array}{l}1,9,21,22,28,30,42,44,47,50,53,54, \\
59,61,62,66,67,69,70\end{array}$ \\
\hline Modo de funcionamento de equipes & 11 & $9,21,22,28,47,48,51,59,67,71,72$ \\
\hline Cuidado ao longo do tempo & 06 & $6,9,16,22,40,70$ \\
\hline $\begin{array}{l}\text { Requisito essencial para a interdisciplinaridade/ } \\
\text { troca de saberes/relações dialógicas }\end{array}$ & 24 & $\begin{array}{l}1,3,6,9,16,19,21,22,30,44,47,48,52, \\
54,55,56,57,60,63,64,65,66,72,73\end{array}$ \\
\hline $\begin{array}{l}\text { Oposição à verticalidade nos serviços e nas } \\
\text { relações }\end{array}$ & 05 & $16,37,44,64,66$ \\
\hline Singularidade do sujeito & 05 & $3,57,58,59,68$ \\
\hline
\end{tabular}

Fonte: elaboração própria. 
graus e tipos de conhecimento, sem submissão a uma hierarquia pré-estabelecida ${ }^{35}$.

Pensar o especialista como alguém que já sabe e que tem as respostas para as angústias da prática, reforça a mitificação sobre o conhecimento, as relações verticais e o foco na patologia que "existe" independente do sujeito. Neste contexto, torna-se difícil exercer a consulta conjunta, prática capital do AM, pois sem horizontalidade esta ferramenta é reduzida a uma mera consulta com o especialista ${ }^{15,16,18,36-38}$ e, assim, perde-se o compartilhamento de competências, a produção de conhecimento e a eficácia da comunicação entre os participantes.

Ressalta-se, portanto, que relações horizontais são afeitas à equidade, enquanto as verticais à hierarquia, confirmando o pensamento de que o modelo tradicional "tende a perpetuar uma relação de poder através da disposição de lugares instituídos de saber e não saber" ${ }^{39}$.

A manutenção das estruturas relacionais verticais é reforçada pela cultura hegemônica que torna privado o saber profissional nuclear, resultando em exclusões de responsabilidade entre os profissionais, enrijecendo fronteiras do saber e estimulando a reserva de mercado ${ }^{7}$.

Outro ponto que merece reflexão é o distanciamento do que se defende por AM e o que de fato isso traduz na prática. Em alguns cenários, o profissional até acredita no modelo da proposta, no entanto, no cotidiano do serviço, ele não a aplica, reforçando a ideia de que a teoria é necessária, mas não suficiente para a práxis ${ }^{17}$.

Para além disso, ainda temos as questões referentes às formações acadêmicas dos profissionais de saúde que, de maneira geral, não apresentam articulação com os princípios do SUS e se mostram insuficientes para o trabalho como apoiadores matriciais ${ }^{16}$.

\section{Sobre supervisão}

Os 29 artigos selecionados que versam sobre o conceito de supervisão oferecem diferentes entendimentos dos quais ressaltam-se:

1) Como prática comum no $\mathrm{AM}$ e em sua maioria em uma relação verticalizada ${ }^{17,31,40,41}$.

Desta forma, pode-se inferir que quando os pSM se propõem a supervisionar os pAPS sem que a proposta de horizontalidade das relações esteja presente, há uma tendência de "orientar" os pAPS, o que não corrobora com a proposta do AM.

Nesta concepção a etimologia latina do termo se apresenta, em que "super" significa "sobre" e visão vem de "visio", visão, ou seja, visão sobre algo ou alguém.

A prática de supervisão em Saúde Mental é tradicional, no entanto, neste caso específico não está em questão a supervisão de um profissional mais experiente com profissionais de SM menos experientes, mas, sim, de profissionais da Saúde Mental exercendo o apoio matricial com profissionais da APS. A "expertise" em Saúde Mental não deve repetir o modelo de supervisão, quando o objetivo na relação entre matriciador e profissional da APS não é transformá-lo em um especialista em SM, e sim colaborar para que este profissional lide com as vicissitudes deste campo dentro de sua própria "expertise".

2) Como supervisão clínico-institucional que se dá para a própria equipe de AM frequentemente associada a um CAPS.

3) Como supervisão técnica matricial, termo usado pressupondo uma horizontalidade implícita nas práticas de saúde mental.

Há ainda duas expressões encontradas em diferentes trabalhos, "supervisão matricial especializada",21 e "supervisão técnica matricial" ${ }^{2}$, as duas utilizadas com o mesmo sentido, ou seja, buscando ampliar os processos de reflexão crítica e de educação permanente e, portanto, compatível com a horizontalidade. O aprofundamento dessa questão encontra-se em um estudo no qual há a defesa de uma interação dialética entre um saber externo com característica ontológica e outro interno relacionado a práxis ${ }^{43}$, ou seja, entre saberes que constituem a formação pessoal e profissional e aqueles que emergem das práticas cotidianas no serviço. Nesse sentido, compreende-se que através do acúmulo dessas competências é possível trabalhar sob a perspectiva de uma "supervisão matricial" que, por sua vez, pode evitar o caráter autoritário e verticalizado inerente à ideia de supervisão já conhecida. Por outro lado, pode-se argumentar a dificuldade que essa possibilidade trás, pois exige conhecimento e reflexão sobre o sujeito, suas relações e o meio no qual sua práxis está inserida. É, portanto, possível supor pela bibliografia estudada, que sob a ótica do AM os significados atribuídos ao conceito de supervisão foram ampliados.

Outra percepção encontrada entre os trabalhos selecionados refere-se ao entendimento dos psicólogos sobre AM, que o compreendiam como discussão de caso e supervisão e se viam com a função de "capacitar" e "orientar" os profissionais que não tinham formação na área de psicologia ${ }^{41}$. Nessa perspectiva, há uma reprodução do modelo vertical entre aqueles que detêm o saber e aqueles que precisam adquirí- $\mathrm{lo}^{17}$. 
O termo Supervisão, portanto, reforça a prática de profissionais, formados nas concepções pedagógicas tradicionais, em que o modelo hierárquico professor-aluno impera, traduzindo o formato bancário do processo ensino-aprendizagem $^{32}$.

A literatura estudada demonstra uma inconsistência nas práticas com experiências desastrosas, que pode ser exemplificada com um relato $^{44}$ em que uma equipe de SF, ao buscar um trabalho compartilhado com uma equipe de um CAPS Álcool e Drogas, nada obteve além de uma aula magistral e orientações genéricas sobre o que deveriam ou não fazer. Fato que corrobora com a premissa que, como demonstram Iglesias e Avellar ${ }^{41}$, em que a falta de compreensão dos profissionais de SM sobre a proposta, põe em cheque o trabalho de AM. Assim pode-se supor que a literatura ainda não esgotou a temática da falta de "clareza", uma vez que os estudos como os de Machado e Camatta ${ }^{45}$ em artigo de revisão demonstram como há falta de compreensão sobre a "real utilização do matriciamento", e os de Lima e Dimenstein ${ }^{46}$ que demonstram sérios entraves no processo de trabalho: vulnerabilidade política na APS; concepções e modelos de práxis heterogêneos; gestores de diferentes níveis que não oferecem suporte; processos irregulares; fluxos na rede de APS que não são acionados; fluxos na rede de APS que não sofrem influência significativa; equipes de SF e equipes de AM com dificuldades relacionais e de agenda; falta de psiquiatras e persistência do modelo ambulatorial de encaminhamentos e marcação de consulta.

As dificuldades, como a falta de clareza, relatadas nas bibliografias associadas a consistência de experiências descritas, parecem ser da ordem da formação verticalizada e dissociada dos profissionais de Saúde, o que exige mais pesquisa sobre as relações entre ensino e verticalidade/horizontalidade. Assim, podemos supor que o modo com que a forma de pensar, sentir e agir no processo formativo é construída, direciona a compreensão dos conceitos de maneira a confirmar uma ótica previamente estabelecida. Neste cenário as propostas de diálogo e horizontalidade ficam fragilizadas ou inalcançáveis. Entre os grandes desafios estão a transformação das matrizes curriculares da graduação e a viabilização da formação pósgraduada para os profissionais que já estão nas redes de assistência ${ }^{17,41,47-49}$.

Ainda que os temas abordados neste artigo não englobem a totalidade dos conceitos de importância para o AM, e o material pesquisado se resuma a interface SM-APS, este trabalho apresenta como fortaleza o intuito de contribuir com a reflexão sobre as práticas de AM, propondo uma discussão sobre os conceitos e a sua polissemia, e chamando a atenção para os processos formativos na área da saúde, além de indicar um caminho para futuras pesquisas.

\section{Conclusões}

A análise das práticas de AM propostas por Gastão Wagner de Sousa Campos e da literatura que lhe seguiu demonstram que o conceito de horizontalidade é vital, e reflete uma postura ideológica democrática, sem o qual o diálogo entre os diferentes atores não alcança seu potencial transformador da realidade assistencial. Embora as questões polissêmicas estejam presentes, os obstáculos da prática se complementam no modelo ainda hegemônico de formação profissional em Saúde, tradicional, hierárquico, uni-profissional e não dialógico que dificulta a integração e colaboração entre as equipes de apoio matricial e da APS e, consequente, resolubilidade e qualidade assistencial.

No que tange a SM é fundamental destacar que a não compreensão do AM, bem como escassez de profissionais da área na APS, pode resultar na prática de uma lógica ambulatorial o que dificulta a realização de um atendimento integral à saúde da população, em especial aos indivíduos em sofrimento psíquico. 


\section{Colaboradores}

LF Chazan trabalhou na concepção, método e pesquisa e na redação final. SLCL Fortes e KR Camargo Junior contribuíram na revisão crítica e na discussão estratégica de elaboração do artigo.

\section{Referências}

1. Oliveira MM, Campos GWS. Apoios matricial e institucional: analisando suas construções. Cien Saude Colet 2015; 20(1):229-238.

2. Chiaverini DH, organizador. Guia prático de matriciamento em Saúde Mental. Brasília: Centro de Estudo e Pesquisa em Saúde Coletiva; 2011.

3. Bonfim IG, Bastos ENE, Góis CWL, Tófoli LF. Apoio matricial em saúde mental na atenção primária à saúde: uma análise da produção científica e documental. Interface (Botucatu) 2013; 17(45):287-300.

4. Mendonça MHM, Martins MIC, Giovanella L, Escorel S. Desafios para gestão do trabalho a partir de experiências exitosas de expansão da Estratégia de Saúde da Família. Cien Saúde Colet 2010; 15 (5):2355-2365.

5. Campos FE, Belisário SA. O Programa de Saúde da Família e os desafios para a formação profissional e a educação continuada. Interface (Botucatu) 2001; 5(9):133-142.

6. Hirdes A. A perspectiva dos profissionais da Atenção Primária à Saúde sobre o apoio matricial em saúde mental. Cien Saúde Colet 2015; 20(2):371-382.

7. Lancetti A, organizador. Saúde Loucura, 7. Saúde mental e saúde da família. 2a ed. São Paulo: Hucitec; 2001.

8. Bastos S, Soares MA. Estudo diagnóstico da atuação das equipes de apoio matricial em Saúde Mental no Programa Saúde da Família na região sudeste da cidade de São Paulo. BIS Bol Inst Saúde 2008; 45:13-16.

9. Campos GWS. Equipes de referência e apoio especializado matricial: um ensaio sobre a reorganização do trabalho em saúde. Cien Saude Colet 1999; 4(2):393403.

10. Campos GWS. Saúde Paidéia. São Paulo: Hucitec; 2003.

11. Brasil. Ministério da Saúde (MS). Secretaria de Atenção à Saúde. Departamento de Atenção Básica. Diretrizes do NASF: Núcleo de Apoio a Saúde da Família. Brasília: MS; 2009.

12. Brasil. Ministério da Saúde (MS). Secretaria de Atenção à Saúde. Departamento de Atenção Básica. Núcleo de Apoio à Saúde da Família. Brasília: MS; 2014.

13. Brasil. Ministério da Saúde (MS). Saúde mental e atenção básica: o vínculo e o diálogo necessários. Brasília: MS; 2003.

14. Brasil. Ministério da Saúde (MS). Portaria no 154, de 24 de janeiro de 2008. Cria os Núcleos de Apoio à Saúde da Família - NASF. Diário Oficial da União; 2008.

15. Fittipaldi ALM, Romano VF, Barros DC. Nas entrelinhas do olhar: Apoio Matricial e os profissionais da Estratégia Saúde da Família. Saúde Debate 2015; 39(104):76-87.

16. Castro CP, Campos GWS. Apoio Matricial como articulador das relações interprofissionais entre serviços especializados e atenção primária à saúde. Physis 2016; 26(2):455-481.

17. Klein AP, d'Oliveira AFPL. O "cabo de força" da assistência: concepção e prática de psicólogos sobre o Apoio Matricial no Núcleo de Apoio à Saúde da Família. Cad Saúde Pública 2017; 33(1):e00158815.

18. Tófoli LF, Fortes S. Apoio matricial de saúde mental na atenção primária no município de Sobral-CE: o relato de experiência. SANARE 2007; 6(2):34-42. 
19. Costa FRM, Lima VV, Silva RF, Fioroni LN. Desafios do apoio matricial como prática educacional: a saúde mental na atenção básica. Interface (Botucatu) 2015; 19(54):491-502.

20. Campos GWS. Um método para análise e co-gestão de coletivos. São Paulo; Hucitec; 2000.

21. Campos GWS. O anti-Taylor: sobre a invenção de um método para co-governar instituições de saúde produzindo liberdade e compromisso. Cad Saúde Pública 1998; 14(4):863-870

22. Campos GWS, Domitti AC. Apoio Matricial e Equipe de Referência: uma metodologia para gestão do trabalho interdisciplinar em saúde. Cad Saude Publica 2007; 23(2):399-408.

23. Brasil. Ministério da Saúde (MS). Secretaria de Atenção à Saúde. Departamento de Atenção Básica. Saúde mental. Brasília: MS; 2013.

24. Cunha GT. A construção da clínica ampliada na atenção básica. São Paulo: Editora Hucitec; 2005.

25. Green BN, Johnson CD, Adams A. Writing narrative literature reviews for peer-reviewed journals: secrets of the trade. Alan Adams. Sports Chiropr Rehabil 2001; 15(1):5-16.

26. Rother ET. Revisão sistemática X revisão narrativa. Acta Paul Enferm 2007; 20(2):V-VI.

27. Cronin P, Ryan F, Coughlan M. Undertaking a literature review: a step-by-step approach. Br J Nurs 2008 17(1):38-43.

28. Barros JO, Gonçalves RMA, Kaltner RP, Lancman S Estratégia do apoio matricial: a experiência de duas equipes do Núcleo de Apoio à Saúde da Família (NASF) da cidade de São Paulo, Brasil. Cien Saúde Colet 2015; 20(9):2847-2856.

29. Merhy EE. Engravidando palavras: o caso da integralidade. In: Pinheiro R.; Mattos RA, organizadores. Construção social da demanda: direito à saúde, trabatho em equipe, participação e espaços públicos. Rio de Janeiro: UERJ-IMS; 2005. p. 195-206.

30. Penido CMF, Alves M, Sena RR, Freitas MIF. Apoio matricial como tecnologia em saúde. Saúde Debate 2010;34(86):467-474.

31. Costa-Rosa A, Luzio CA, Yasui S. As Conferências Nacionais de Saúde Mental e as premissas do modo psicossocial. Saúde Debate 2001; 25(58):12-25.

32. Freire P. Pedagogia do Oprimido. $17^{\mathrm{a}}$ ed. Rio de Janeiro: Paz e Terra; 1987.

33. Severo AK, Dimenstein M. Processos de trabalho e gestão na estratégia de atenção psicossocial. Psicol Soc 2011;23(2):340-349.

34. Barreto A. Terapia comunitária: um valor estratégico para a gestão da saúde da comunidade [arquivo de áudio na Internet]. [acessado 2016 Out 02]. Disponível em http://www5.ensp.fiocruz.br/biblioteca/home/ exibedetalhesBiblioteca.cfm?ID $=4900 \&$ Tipo $=B$

35. Keim EJ. Princípios Essenciais na Obra Freiriana e a Educação Inter-étinica da Emancipação e Humanização. In: IX Anped Sul 2012, 2012, Caxias do Sul. IX Anped Sul 2012 Seminário de Pesquisa em Educação da Região Sul. Caxias do Sul: Universidade de Caxias do Sul; 2012.
36. Athié K, Fortes S, Delgado PG. Matriciamento em saúde mental na Atenção Primária: uma revisão crítica (2000-2010). Rev Bras Med Fam Com 2013; 8(26):64-74.

37. Cela M, Oliveira IF. O psicólogo no Núcleo de Apoio à saúde da Família: articulação de saberes e ações. Estudos Psicol (Natal) 2015; 20(1):31-39.

38. Fortes S, Menezes A, Athié K, Chazan LF, Rocha H, Thiesen J, Ragoni C, Pithon T, Machado A. Psiquiatria no século XXI: transformações a partir da integração com a Atenção Primária pelo matriciamento. Physis 2014; 24(4):1079-1102.

39. Triandis HC, Gelfand MJ. Converging measurement of horizontal and vertical individualism and collectivism. J Pers Soc Psychol 1998; 74(1):118-128.

40. Dimenstein M, Severo AK, Brito M, Pimenta AL, Medeiros V, Bezerra E. O apoio matricial em Unidades de Saúde da Família: experimentando inovações em saúde mental. Saude Soc 2009; 18(1):63-74.

41. Iglesias A, Avellar LZ. As Contribuições dos Psicólogos para o Matriciamento em Saúde Mental. Psicol Cien Prof 2016; 36(2):364-379.

42. Cunha GT, Campos GWS. Apoio Matricial e Atenção Primária em Saúde. Saude Soc 2011; 20(4):961-970.

43. Campos GWS. Um Método de Análise e Co-gestão de coletivos. A constituição do sujeito, a produção de valor de uso e a democracia em instituiçães: O Método da Roda. São Paulo: Hucitec; 2000

44. Medeiros RHA. Uma noção de matriciamento que merece ser resgatada para o encontro colaborativo entre equipes de saúde e serviços no SUS. Physis 2015; 25(4):1165-1184.

45. Machado DKS, Camatta MW. Apoio matricial como ferramenta de articulação entre a Saúde Mental e a Atenção Primária à Saúde. Cad Saúde Colet 2013; 21(2):224-232.

46. Lima M, Dimenstein M. O apoio matricial em saúde mental: uma ferramenta apoiadora da atenção à crise. Interface (Botucatu) 2016; 20(58):625-635.

47. Gryschek G, Pinto AAM. Saúde Mental: como as equipes de Saúde da Família podem integrar esse cuidado na Atenção Básica? Cien Saúde Colet 2015; 20(10):3255-3262.

48. Bispo Júnior JP, Moreira DC. Educação permanente e apoio matricial: formação, vivências e práticas dos profissionais dos Núcleos de Apoio à Saúde da Família e das equipes apoiadas. Cad Saúde Pública 2017; 33(9):e00108116.

49. Reis ML, Medeiros M, Pacheco LR, Caixeta CC. Evaluation of the multiprofessional work of the family health support center (NASF). Texto Contexto Enferm 2016; 25(1):e2810014.

50. Figueiredo MD, Campos RO. Saúde Mental na atenção básica à saúde de Campinas, SP: uma rede ou um emaranhado? Cien Saúde Colet 2009; 14(1):129-138.

51. Soares M. A inserção do enfermeiro psiquiátrico na equipe de apoio matricial em saúde mental. SMAD Rev Eletr Saúde Ment Álcool Drog 2008; 4(2):1-4.

52. Böing E, Crepaldi MA. O psicólogo na atenção básica: uma incursão pelas políticas públicas de saúde brasileiras. Psicol Cien Prof 2010; 30(3):634-649. 
53. Sousa FSP, Jorge MSB, Vasconcelos MGF, Barros MMM, Quinderé PHD, Gondim LGF. Tecendo a rede assistencial em saúde mental com a ferramenta matricial. Physis 2011;21(4):1579-1599.

54. Rosa FM, Weiller TH, François APW, Brites LS, Silveira D, Righi LB. O olhar das equipes de referência sobre o trabalho realizado pelo apoio matricial. Rev Enferm UFSM 2011; 1(3):377-385.

55. Cavalcante CM, Pinto DM, Carvalho AZT, Jorge MSB, Freitas CHA. Desafios do cuidado em saúde mental na estratégia saúde da família. RBPS 2011; 24(2):102108.

56. Cossetin A, Olschowsky A. Avaliação das ações em saúde mental na estratégia de saúde da família: necessidades e potencialidades. Rev Gaúcha Enferm 2011; 32(3):495-501.

57. Morais APP, Tanaka OU. Apoio matricial em saúde mental: alcances e limites na atenção básica. Saude Soc 2012; 21(1):161-170.

58. Pinto AGA, Jorge MSB, Vasconcelos MGF, Sampaio JJC, Lima GP, Bastos VC, Sampaio HAC. Apoio matricial como dispositivo do cuidado em saúde mental na atenção primária: olhares múltiplos e dispositivos para resolubilidade. Cien Saude Colet 2012; 17(3):653660.

59. Jorge MSB, Sousa FSP, Franco TB. Apoio matricial: dispositivo para resolução de casos clínicos de saúde mental na Atenção Primária à Saúde. Rev Bras Enferm 2013; 66(5):738-744.

60. Leal BM, Antoni C. Os Centros de Atenção Psicossocial (CAPS): estruturação, interdisciplinaridade e intersetorialidade. Aletheia 2013; 40:87-101.

61. Casanova AO, Teixeira MB, Montenegro E. O apoio institucional como pilar na cogestão da atenção primária à saúde: a experiência do Programa TEIAS - Escola Manguinhos no Rio de Janeiro, Brasil. Cien Saude Colet 2014; 19(11):4417-4426.

62. Moura RH, Luzio CA. O apoio institucional como uma das faces da função apoio no Núcleo de Apoio à Saúde da Família (NASF): para além das diretrizes. Interface (Botucatu) 2014; 18(Supl. 1):957-970.

63. Fonseca SD, Machado ATGM, Lima AMLD, Jorge AO, Reis CMR, Abreu DMX, Araújo LHL, Evangelista MLF, Escobar AL, Santos CRI, Santos AF. Compreendendo o apoio matricial e o resultado da certificação de qualidade nas áreas de atenção à criança, mulher, diabetes/hipertensão e saúde mental. Saúde Debate 2014; 38(esp.):83-93.

64. Pegoraro RF, Cassimiro TJL, Leão NC. Matriciamento Em Saúde Mental Segundo Profissionais Da Estratégia Da Saúde Da Família. Psicol Estud 2014; 19(4):621631.

65. Campos GWS, Figueiredo MD, Pereira Junior N, Castro CP. A aplicação da metodologia Paideia no apoio institucional, no apoio matricial e na clínica ampliada. Interface (Botucatu) 2014; 18(Supl. 1):983-995.

66. Paixão L, Tavares ML. A construção do projeto "Apoio de rede" como estratégia institucional. Interface (Botucatu) 2014; 18(Supl. 1):845-858.

67. Hirdes A, Silva MKR. Apoio matricial: um caminho para a integração saúde mental e atenção primária. Saúde Debate 2014; 38(102):582-592.
68. Jorge MSB, Vasconcelos MGF, Maia Neto JP, Gondim LGF, Simões ECP, Simões E. Possibilidades e desafios do apoio matricial na atenção básica: percepções dos profissionais. Rev Psicol Teor Prática 2014; 16(2):6374.

69. Gazignato ECS, Silva CRC. Saúde mental na atenção básica: o trabalho em rede e o matriciamento em saúde mental na Estratégia de Saúde da Família. Saúde Debate 2014; 38(101):296-304.

70. Sampaio J, Melo MC, Grigório MC, Soares RS. Processos de Trabalho dos Núcleos de Atenção à Saúde da Família junto a Atenção Básica: Implicações para a articulação de Redes Territoriais de Cuidado em Saúde. Rev Bras Cien Saúde 2015; 19(1):41-48.

71. Hirdes A, Scarparo HBK. O labirinto e o minotauro: saúde mental na Atenção Primária à Saúde. Cien Saude Colet 2015; 20(2):383-393.

72. Matuda CG, Pinto NRS, Martins CL, Frazão P. Colaboração interprofissional na Estratégia Saúde da Família: implicações para a produção do cuidado e a gestão do trabalho. Cien Saude Colet 2015; 20(8):2511-2521.

73. Tesser CD. Núcleos de Apoio à Saúde da Família, seus potenciais e entraves: uma interpretação a partir da atenção primária à saúde. Interface (Botucatu) 2017; 21(62):565-578.

74. Bezerra E, Dimenstein M. Os CAPS e o trabalho em rede: tecendo o apoio matricial na atenção básica. $P s i-$ col Cien Prof 2008; 28(3):632-645.

75. Dimenstein M, Galvão VM, Severo AKS. O Apoio Matricial na Perspectiva de Coordenadoras de Equipes. Pesqui Prát Psicossociais 2009; 4(1):37-48.

76. Ballarin MLGS, Carvalho FB, Ferigato SH, Miranda IMS, Magaldi CC. Centro de atenção psicossocial: convergência entre saúde mental e coletiva. Psicol Estud 2011; 16(4):603-611.

77. Belloti M, Lavrador MCC. Apoio matricial: cartografando seus efeitos na rede de cuidados e no processo de desinstitucionalização da loucura. P e Psique 2012; 2(3):128-146.

78. Azevedo DM, Guimarães FJ, Dantas JF, Rocha TM. Atenção básica e saúde mental: um diálogo e articulação necessários. Rev APS 2014; 17(4):537-543.

79. Neto MRG, Medina STS, Hirdes MA. Apoio matricial em saúde mental na percepção dos profissionais especialistas. Aletheia 2014; 45:139-155.

80. Moura RFS, Silva CRC. Saúde Mental na Atenção Básica: Sentidos Atribuídos pelos Agentes Comunitários de Saúde. Psicol Cien Prof 2015; 35(1):199-210.

81. Castro CP, Oliveira MM, Campos GWS. Apoio matricial no SUS Campinas: análise da consolidação de uma prática interprofissional na rede de saúde. Cien Saude Colet 2016; 21(5):1625-1636.

Artigo apresentado em 05/04/2018

Aprovado em 03/12/2018

Versão final apresentada em $05 / 12 / 2018$ 